This is an electronic reprint of the original article. This reprint may differ from the original in pagination and typographic detail.

\author{
Author(s): Nonappa; Lahtinen, Tanja; Haataja, Johannes S.; Tero, Tiia-Riikka; Häkkinen, Hannu; \\ Ikkala, Olli
}

Title: $\quad$ Template-Free Supracolloidal Self-Assembly of Atomically Precise Gold Nanoclusters : From 2D Colloidal Crystals to Spherical Capsids

Year: $\quad 2016$

Version:

Please cite the original version:

Nonappa, Lahtinen, Tanja, Haataja, Johannes S., Tero, Tiia-Riikka, Häkkinen, Hannu, Ikkala, Olli. (2016). Template-Free Supracolloidal Self-Assembly of Atomically Precise Gold Nanoclusters : From 2D Colloidal Crystals to Spherical Capsids. Angewandte Chemie International Edition, 55(52), 16035-16038.

https://doi.org/10.1002/anie.201609036

All material supplied via JYX is protected by copyright and other intellectual property rights, and duplication or sale of all or part of any of the repository collections is not permitted, except that material may be duplicated by you for your research use or educational purposes in electronic or print form. You must obtain permission for any other use. Electronic or print copies may not be offered, whether for sale or otherwise to anyone who is not an authorised user. 


\title{
Template-free supracolloidal self-assembly of atomically precise gold nanoclusters: From 2D colloidal crystals to spherical capsids
}

\author{
Nonappa $^{*[a]}$, Tanja Lahtinen ${ }^{[b]}$, Johannes. S. Haataja ${ }^{[a]}$, Hannu Häkkinen ${ }^{*[b]}$, and Olli Ikkala ${ }^{*[a]}$
}

\begin{abstract}
We report supracolloidal self-assembly of atomically precise and strictly monodisperse gold nanoclusters involving $p$ mercaptobenzoic acid ligands $\left(A u_{102}-p M B A_{44}\right)$ under aqueous conditions into hexagonally packed monolayer thick 2-dimensional facetted colloidal crystals (thickness $2.7 \mathrm{~nm}$ ) and their bending to closed shells leading to spherical capsids (d $\sim 200 \mathrm{~nm})$, as controlled by solvent conditions. The $2 D$ colloidal assembly is driven in template-free manner by the spontaneous patchiness of the $p M B A$ ligands around the $A u_{102}-p M B A_{44}$ nanoclusters preferably towards equatorial plane, thus promoting inter-nanocluster hydrogen bonds and high packing to planar sheets. More generally, the findings encourage to explore atomically precise nanoclusters towards highly controlled colloidal self-assemblies.
\end{abstract}

In materials science there is a need for well-defined selfassembled structures bridging the length scales upon pursuing new functionalities. ${ }^{[1]}$ This requires mastering the size and structural uniformities of the building blocks, as well as the complex interplay of competing interactions. ${ }^{[2-5]}$ In supramolecular chemistry of small molecules, atomically precise molecular structures and controlled supramolecular interactions are readily available, thus allowing detailed engineering of the molecular packings. For progressively larger structural units, achieving strictly defined sizes and interactions becomes more challenging. Polymers can lead to hierarchical self-assemblies, provided they involve sufficiently narrow molecular weights distributions. ${ }^{[2,7]}$ Whereas dendrimers and dendrons provide precision for structural control, ${ }^{[6]}$ achieving a monodispersity of high polymers involves challenges. However, that monodispersity can lead to fundamentally different polymeric self-assembly has been directly shown. ${ }^{[8]}$ In the next larger length scale, colloids have allowed superlattices, superparticles, colloidosomes, protein-like assemblies, patchy particles, directional colloids with "valence", and colloidal chains. ${ }^{[9-17]}$ However, achieving strictly defined colloidal building blocks for self-assemblies is highly challenging. ${ }^{[18]}$ That a monodispersity of colloidal units and the underlying controlled interactions could lead to novel assemblies, is exemplified by co-assemblies of icosahedral viruses with small gold nanoparticles, where the

[a] Prof. O. Ikkala, Dr. Nonappa, M.Sc. J. S. Haataja Department of Applied Physics, Molecular Materials Group Aalto University School of Science

Puumiehenkuja 2, Espoo, FI-02150, Finland

E-mail:olli.ikkala@aalto.fi, nonappa@aalto.fi

[b] Prof. H. Häkkinen, Dr. T. Lahtinen

Department of Chemistry and Physics, Nanoscience centre University of Jyväskylä,

Survontie 9, FI-40014, Jyväskylä, Finland

email: hannu.j.hakkinen@jyu.fi

Supporting information for this article is given via a link at the end of the document.((Please delete this text if not appropriate)) monodispersity and the interactions of the viral capsids control the colloidal crystalline packing. ${ }^{[19]}$ The above considerations motivate to explore the potential self-assemblies using strictly monodisperse colloidal particles.

However, there are no reports on the self-assembly atomically precise synthetic nanoparticles into colloidal superstructures. This is surprising, as there has been significant progress in the synthesis and characterization of such gold and silver nanoclusters. ${ }^{[20,21]}$ Their internal structures have been characterized using X-ray cystallography, cryo-transmission electron microscopy (cryo-TEM), mass spectroscopy, as combined with molecular dynamics (MD) simulations. ${ }^{[22,23]}$ Therefore, they could be feasible building blocks to study colloidal self-assemblies. In particular, $\mathrm{Au}_{102}-\mathrm{pMBA}_{44}$ containing $102 \mathrm{Au}$-atoms and $44 \mathrm{pMBA}$-ligands was the first nanocluster whose solid state structure was solved in detail using X-ray crystallography. ${ }^{[22]}$ Recent studies the NMR spectra for ligand layer of $\mathrm{Au}_{102}-\mathrm{pMBA}_{44}$ has been fully assigned in water using $2 \mathrm{D}$ NMR measurements. This study in combination with dynamical MD simulations confirmed that the ligand layer arrangement in solution state is similar to that of solid state structure. ${ }^{[24]}$

We envisaged that $\mathrm{Au}_{102}-\mathrm{pMBA}_{44}$ (Figure 1) with its peripheral carboxylic acid groups of pMBA, allowing hydrogen bonds between nanoclusters, provides a feasible starting point to investigate colloidal superstructures using atomically precise building blocks. When the carboxylic acids are in the acid form (protonated), $\mathrm{Au}_{102}-\mathrm{pMBA}_{44}$ is soluble in polar solvents such as methanol and dimethyl sulfoxide and insoluble in water. ${ }^{[25]}$ However, upon deprotonating a fraction of carboxylic acids (>22) into sodium salts using aqueous sodium hydroxide, the nanoclusters become dispersible in water. ${ }^{[25 a]}$ However, these partially deprotonated water-dispersible clusters tend to precipitate upon adding methanol. Further, the nanoclusters are methanol-soluble when the number of deprotonated carboxylic groups are between 0-7 and beyond that the clusters precipitate in methanol. Importantly for the present work, have shown that $\mathrm{Au}_{102}-\mathrm{pMBA}_{44}$ is not completely spherical, and that there exists patchiness of neutral carboxylic acid groups and the deprotonated negative carboxylate groups. ${ }^{[25 a]}$ Depending on the solvent conditions, here we show that $\mathrm{Au}_{102}-\mathrm{pMBA}_{44}$ nanoclusters allow tunable colloidal self-assemblies to $2 \mathrm{D}$ nanosheets of monoparticle thickness and their curvature to closed shells (capsids).

The $\mathrm{Au}_{102}-\mathrm{pMBA}_{44}$ nanoclusters were synthesized from $\mathrm{HAuCl}_{4} 3 \mathrm{H}_{2} \mathrm{O}$ under sodium borohydride reduction in the presence of $p$-mercaptobenzoic acid according to the reported procedure (Figure 1a, see also Supporting Information). ${ }^{[25 b]}$ The nanoclusters were purified and subsequently characterized using cryo-TEM and mass spectroscopy (Supporting Information, Figures S1\&S2). 
We first dispersed $\mathrm{Au}_{102}-p \mathrm{MBA}_{44}$ in water using aqueous sodium hydroxide $(1.0 \mathrm{M})$, which results in partial deprotonation of carboxylic acids to salt form (Supporting Information). The surface negative charge was confirmed by the zeta $(\zeta)$ potential of $-69 \mathrm{mV}$ (Supporting Information, Figure S3). The TEM micrographs shows well separated aq. individual nanoclusters (Figure 1f).

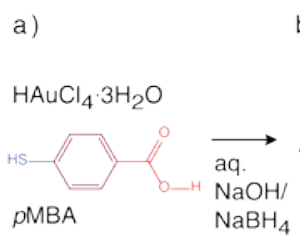

d)

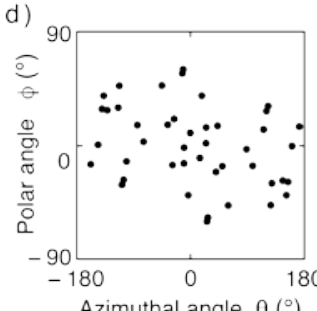

C)

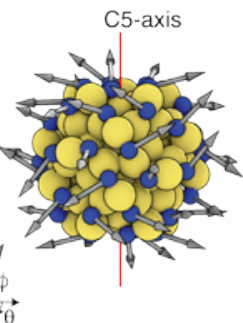

e

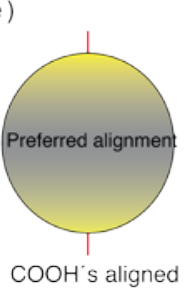

f)

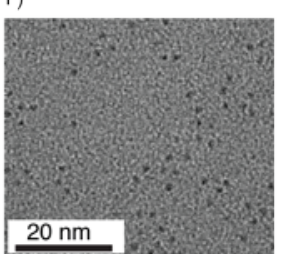

Figure 1. a) Synthetic route for the $\mathrm{Au}_{102}-\mathrm{pMBA}_{44}$ nanoclusters and b) their internal structure extracted from the solid state structure. ${ }^{30} \mathrm{c}$ ) The resolved ligand directions illustrated using arrows, elucidating the possible hydrogen bonding directions. d) A polar coordinate system (polar angle $\phi$ and azimuthal angle $\theta$ ) for $\mathrm{Au}_{102}-\mathrm{pMBA}_{44}$ can be identified where the ligand directions are predominantly in the equatorial plane, as schematically illustrated in (e), thus promoting layered sheets by directional hydrogen bonds predominantly inplane directions. d) TEM micrograph of nanoclusters dispersed in water.

To trigger hydrogen bondings between the $\mathrm{Au}_{102}-p \mathrm{MBA}_{44}$ nanoclusters based on pMBA-mediated carboxylic acid dimerizations, the above aqueous dispersions were dialysed against methanol for 12 hours (Supporting Information). TEM micrographs revealed facetted sheet-like structures (Figure 2a shows 3 stacked layers, see also Supporting Information, Figure S4). At the edges, monolayer sheets with hexagonal internal packings are observed with a repeat unit distance of $2.7 \mathrm{~nm}$ and periodicity of $2.3 \mathrm{~nm}$. This matches with the sizes of individual $\mathrm{Au}_{102}-\mathrm{pMBA}_{44}$ nanoclusters. Therefore, 2D colloidal crystallization of $\mathrm{Au}_{102}-\mathrm{pMBA}_{44}$ nanoclusters to facetted nanosheets of one nanoparticle thickness can be concluded. Unlike the previously reported layered self-assemblies of nanoparticles templated on a substrate upon solvent casting, the present nanosheets form spontaneously without any template. Due to the extended lateral sizes, the sheets have a tendency to stack into layers. This can be promoted by continuing the methanol dialysis (Figures 3a,b, Supporting information Figures S5-S7).

That monolayer 2D colloidal crystal sheets are formed spontaneously in template-free manner is subtle and calls for explanations. The facetted outer rims also support near perfect hexagonal closed packing (hcp) order, facilitated by the monodispersity. That 2D-structures are formed is explained by the ligand arrangement, as the $\mathrm{Au}_{102}-\mathrm{pMBA}_{44}$ nanoclusters are not exactly spherical. Figure $1 \mathrm{~b}$ depicts the resolved internal structure, showing the positions of individual atoms using X-ray diffraction. The directions of carboxylic acids are illustrated by arrows (Figure 1c). The figure gives a qualitative hint that the directions are not distributed equally over the sphere, i.e. there is patchiness, as reported for solid state structure. ${ }^{[22]}$ More quantitavely, Figure 1d shows that it is possible to select a polar coordinate system for a $\mathrm{Au}_{102}-\mathrm{pMBA}_{44}$ nanocluster so that the pMBA-based carboxylic acids are prominently directed to the equatorial plane, with only few ones pointing toward the polar directions, thus breaking the spherical symmetry. Within the azimuthal angles, there is approximately constant density. This suggests that there is an equatorial belt of hydrogen bonding carboxylic acids, towards nanocluster packing as $2 \mathrm{D}$ assemblies. $\mathrm{Hcp}$ is natural to expect due to enthalpic reasons to maximize the hydrogen bondings within the planes.

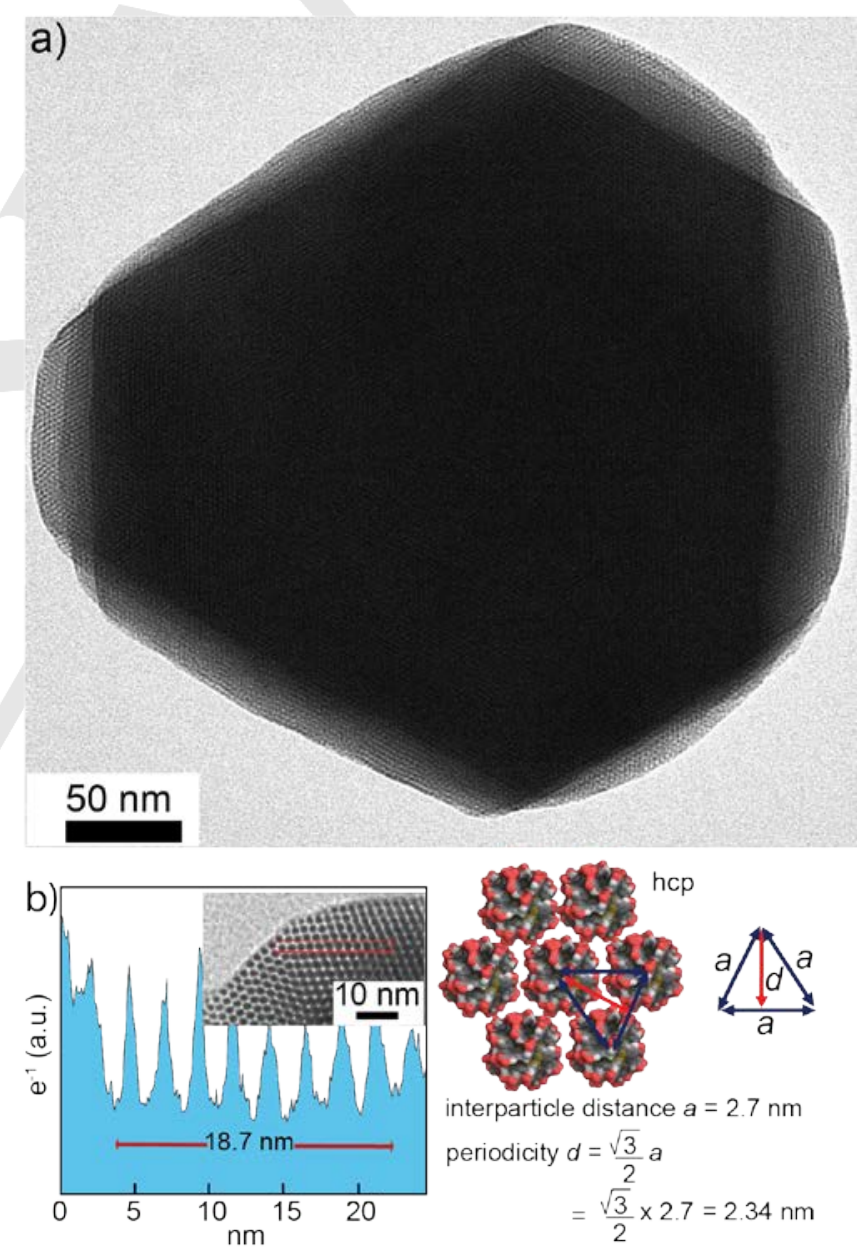

Figure 2. Colloidal crystallization of aq. $A_{102}-p M A_{44}$ nanoclusters into $2 \mathrm{D}$ nanosheets. a) A TEM micrograph of $2 \mathrm{D}$ nanosheets, with facetted edges. Three sheets are stacked. b) A close-up of a monolayer involving near perfect hexagonal closed packed lattice of $\mathrm{Au}_{102}-\mathrm{pMBA}_{44}$ nanoclusters.

As the $\mathrm{Au}_{102}-\mathrm{pMBA}_{44}$ nanoclusters involve less carboxylic acid hydrogen bonding directionality towards the poles than 
towards the equatorial plane (see schematically Figure 1e), some amphiphilic character could be anticipated. One could ask whether closed vesicular structures could be obtained.
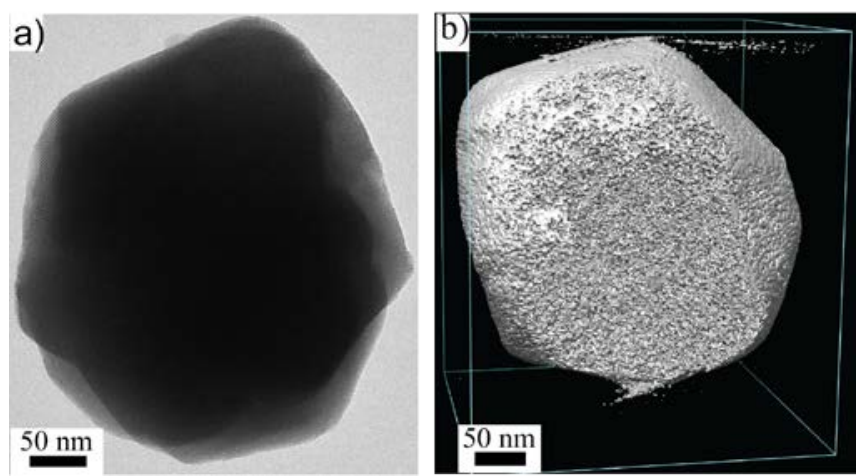

Figure 3. Growing thicker aq. assemblies of $\mathrm{Au}_{102}-\mathrm{pMBA}_{44}$ nanoclusters towards $3 \mathrm{D}$ layered structures by stacking of $2 \mathrm{D}$ colloidal sheets upon continued dialysis against methanol (>12 h). a) TEM. b) Electron tomographic reconstruction.

Indeed, upon adding $10 \mu \mathrm{L}$ of $0.5 \mathrm{mg} / \mathrm{mL}$ nanocluster dispersion into $500 \mu \mathrm{L}$ of methanol furnished spherical superstructures, with an average diameter of $200 \mathrm{~nm}$ as revealed by cryo-TEM (Figure 4a). The presence of spherical superstructures in solution was confirmed using dynamic light scattering, which showed an average size of $220 \mathrm{~nm}$ (Supporting Information, Figure S9). The conventional TEM represents the 2D projections with limited information on the 3D structural details. Therefore, we collected the tilt series between $+69^{\circ}$ to $-69^{\circ}$ with an increment of $2^{\circ}$ between the images (Supporting Information). The alignment using fiducial gold markers followed by 3Ddimensional reconstruction confirmed the spherical nature of superstructures (Figure 4b, Video S1). More detailed analysis of the electron tomograms revealed a uniform shell of a monolayer thickness (Figure 4c), even if the exact positions of the nanoclusters cannot be resolved. Because of the monodispersity of building blocks, we refer these spherical superstructures as capsids, in analogy to virus capsids (which also are composed of monodisperse subunits). That the shell is of monolayer thickness becomes of no surprise based on the above detailed studies of the 2D planar sheets.
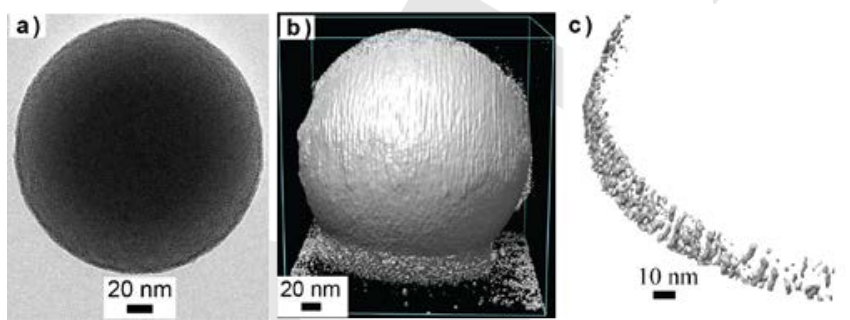

Figure 4. Spherical capsids of aq. $\mathrm{Au}_{102}-\mathrm{pMBA}_{44}$ nanoclusters. a) TEM micrograph. b) Electron tomographic reconstruction and c) a close-up thereof showing monolayer thick shell.
Finally, the pMBA-based hydrogen bonds manifest as possibility to join different capsids. Figure 5 shows higher order assemblies of 2 or 3 capsids as mediated by partial fusion. This paves ways for higher order manipulation of the hollow capsids, for example for lightweight framework materials.
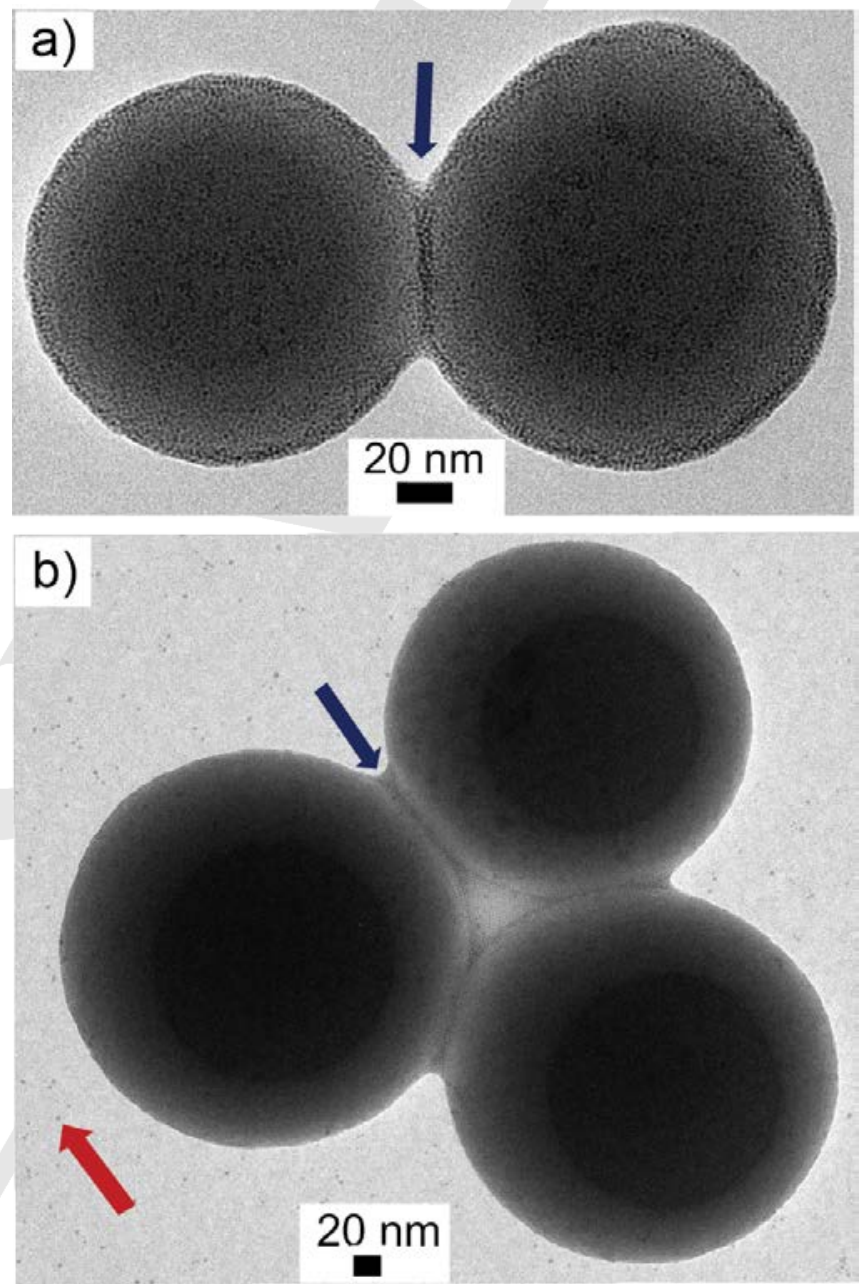

Figure 5. TEM micrographs illustrating pMBA mediated binding between capsids of aq. $\mathrm{Au}_{102}-\mathrm{pMBA}_{44}$ nanoclusters. a) A capsid dimer. b) A capsid trimer. Blue arrows indicate intercapsid hydrogen bonding and red arrows showing the fiducial gold nanoparticles $(5 \mathrm{~nm})$.

In conclusion, by using aqueous $\mathrm{Au}_{102}-\mathrm{pMBA}_{44}$ as a model nanocluster system, we have shown that strictly monodisperse and atomically precise gold nanoclusters are potential building blocks for template-free 2D nanosheets and closed spherical capsids as promoted by the inherent and spontaneous nonspherical arrangement of the hydrogen bonding pMBAligands promoting planar packing. Such a colloidal selfassembly is tuned by controlling the protonation/deprotonation balance of the carboxylic acids, where the protonated form facilitates intercluster hydrogen bonds and the deprotonated carboxylate salt form promotes water dispersibility. The concept can be useful to prepare novel $2 \mathrm{D}$ materials with additional properties such as plasmonic functionalities, novel mechanical 
properties and even flexibility by introducing dynamic supramolecular or covalent intercluster bondings, or controlled stacking to $3 \mathrm{D}$ crystals. With increasing knowledge on the synthesis of well-defined nanoclusters, we believe that the present work will encourage both nanocluster and colloidal community to investigate new clusters as colloidal building blocks. We suggest that the present findings are, in fact, more general, as it may turn out that several types of strictly monodisperse nanoclusters turn out not to be fully spherically symmetric and this broken symmetry can be used for benefit to construct higher order colloidal assemblies.

\section{Acknowledgements}

Funding from Academy of Finland for academy professorship (O.I. and $\mathrm{H}$. H), Centre of Excellence in Molecular Engineering of Biosynthetic Hybrid Materials (HYBER, 2014-2019), and ERC Advanced Grant MIMEFUN (2012-2017) are acknowledged. This work made use of the CSC-IT centre (Espoo) computational facilities and Aalto University Nanomicroscopy Center (Aalto-NMC) premises.

Keywords: Gold nanoclusters $\cdot$ colloidal self-assembly $\cdot 2 \mathrm{D}$ colloidal crystal $\bullet$ hydrogen bond $\bullet$ patchy

\section{References}

[1] G. M.Whitesides, B. Grzybowski, Science, 2002, 295, 2418-2421.

[2]. O. Ikkala, N. Houbenov, P. Rannou in Handbook of Liquid Crystals, Vol 8, eds. J. W. Goodby, P. J. Collings, T. Kato, C. Tschierske, H. Gleeson, P. Raynes, Wiley-VCH, Weinheim, 2014.

[3]. K. Harris, D. Fujita, M. Fujita, Chem. Commun., 2013, 49, 6703-6712.

[4]. T. Aida, E.W. Meijer, S. I. Stupp, Science, 2012, 335, 813-817.

[5]. P. Prasenjit Mal, B. Breiner, K. Rissanen, J. R. Nitschke, Science, 2009, 324, 1696-1699.

[6]. V. Percec, D. A. Wilson, P. Leowanawat, C. J. Wilson, A. D. Hughes, M. S. Kaucher, D. A. Hammer, D. H. Levine, A. J. Kim, F. S. Bates, K. P Davis, T. P. Lodge, M. L. Klein, R. H. DeVane, E. Aqad, B. M.Rosen, A O. Argintaru, M. J. Sienkowska, K. Rissanen, S. Nummelin, J. Ropponen, Science, 2010, 328, 1009-1014.

[7]. V. Abetz, P. F. W. Simon, Adv. Polym. Sci, 2005, 189, 125-212.

[8]. S. M. Yu, V. P. Conticello, G. Zhang, C. Kayser, M. J. Fournier, T. L. Mason, D. A. Tirrell, Nature, 1997, 389, 167-170.
[9]. C. P. Collier, T. Vossmeyer, J. R. Heath. Annu Rev. Phys. Chem. 1998 49, 371-404.

[10]. Q. Chen, S. C. Bae, S. Granick, Nature, 2011, 469, 381-384.

[11]. N. A. Kotov. Science, 2010, 310, 188-189.

[12]. T. Wang, J. Zhuang, J. Lynch, O. Chen, Z. Wang, X. Wang, D. LaMontagne, H. Wu, Z. Wang, Y. C. Cao, Science, 2012, 338, 358-363.

[13]. A. H. Gröschel, A. Walther, T. Löbling, F. H. Schacher, H. Schmalz, A. H. Müller, Nature, 2013, 503, 247-251.

[14]. Y. Wang, Y. Wang, D. R. Breed, V. N. Manoharan, L. Feng, A. D. Hollingsworth, M. Weck, D. J. Pine, Nature, 2012, 491, 51-56.

[15]. Z. Nie, D. Fava, E. Kumacheva, S. Zou, G. C. Walker, M. Rubinstein, Nature Mater. 2007, 6, 609-614.

[16]. J. Zeng, J. Huang, W. Lu, X. Wang, B. Wang, S. Zhang and J. Hou Adv. Mater. 2007, 19, 2172-2176.

[17]. R. Erb, H. S. Son, B. Samanta, V. M. Rotello, B. B. Yellen. Nature 2009, 457, 999-1002.

[18]. V. N. Manoharan, Science 2015, 349, 1253751.

[19]. a) M. A. Kostiainen, P. Hiekkataipale, A. Laiho, V. Lemieux, J. Seitsonen, J. Ruokolainen, P. Ceci, Nature Nanotechnology, 2013, 8, 52-56; b) V. Liljeström, J. Mikkilä, M. Kostiainen, Nature Commun. 2014, 5, Article number:4445doi:10.1038/ncomms5445.

[20]. H. Häkkinen, Nature Chem. 2012, 4, 443-455;

[21]. a) b) R. Jin, Nanoscale. 2015, 7,1549-65; b) R. Jin, C. Zeng, M. Zhou, Y. Chen, Chem. Rev., 2016, DOI: 10.1021/acs.chemrev.5b00703

[22]. P. D. Jadzinksy, G. Calero C. J. Ackerson, D. A. Bushnell and R. D. Kornberg, Science, 2007, 318, 430-433.

[23] M. Azubel, J. Koivisto, S. Malola, D. Bushnell, G. L. Hura, A.L. Koh, H. Tsunoyama, T. Tsukuda, M. Pettersson, H. Häkkinen, R. D. Kornberg, Science, 2014, 345, 909.

[24] K. Salorinne, S. Malola, O. A. Wong, C. D. Rithner, X. Chen, C. J. Ackerson, H. Häkkinen, Nature Commun., 2015, 7, Article number:10401, doi:10.1038/ncomms10401.

[25]. a) J. Koivisto, X. Chen, S. Donnini, T. Lahtinen, H. Häkkinen, G. Groenhof, M. Pettersson, J. Phys. Chem. C, 2016, 120, 10041-10050; b) K. Salorinne, T. Lahtinen, S. Malola, J. Koivisto, H. Häkkinen, Nanoscale, 2014, 6, 7823-7826; c) M. Martikainen, K. Salorinne, T. Lahtinen, S. Malola, P. Permi, H. Häkkinen, V. Marjomäki, Nanoscale, 2015, 7, 17457-17467. d) C. L. Heinecke, T.W. Ni, S.A. Malola, V.P. Mäkinen, O.A. Wong, H. Häkkinen, C. J. Ackerson, J. Am. Chem. Soc. 2012, 134, 13316-13322; e) M. Walter, J. Akola, O. LopezAcevedo, P. D. Jadzinsky, G. Calero, C. J. Ackerson, R. L. Whetten, H. Grönbeck, H. Häkkinen, Proc. Natl. Acad. Sci. 2008, 105, 9157. 
Entry for the Table of Contents (Please choose one layout)

\section{COMMUNICATION}

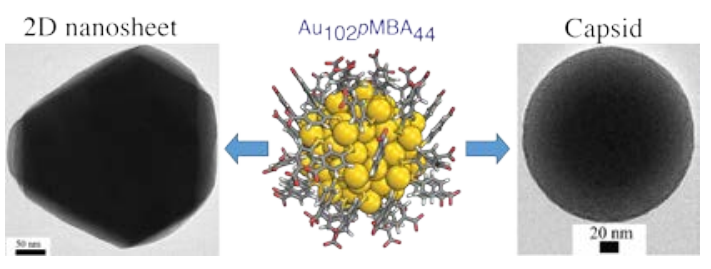

Colloidal self-assembly of atomically precise, monodisperse gold nanoclusters to $2 \mathrm{D}$ colloid crystals and their formation of closed shells with monolayer thick shells are presented.
Nonappa*, T. Lahtinen, J. S. Haataja, $H$. Häkkinen*, O. Ikkala*

Page No. - Page No.

Template-free supracolloidal selfassembly of atomically precise gold nanoclusters: From 2D colloidal crystals to spherical capsids 